\title{
Original Article: Prognostic Factors of Recurrence Pattern, Survival and Toxicity in Patients with Gastric Cancer Treated According to Protocol INT-0116 with 3D-Conformal Radiotherapy
}

\author{
Guilherme Rocha Melo Gondim*, Ricardo Cesar Fogaroli, Antonio Cassio Assis \\ Pellizzon, Douglas Guedes de Castro, Maria Leticia Gobo Silva, Michael Jenwei \\ Chen, Marcel Davi Loureiro de Melo \\ Radiation Oncology Department, AC Camargo Cancer Center, Sao Paulo, Brazil
}

Received: September 02, 2015; Accepted: December 04, 2015; Published: December 18, 2015

*Corresponding author: Guilherme Rocha Melo Gondim, Radiation Oncology Department, AC Camargo Cancer Center, Rua Professor Antonio Prudente, 211 Liberdade, Sao Paulo, SP. Brazil. Phone: +55(11)99888-8830; E-mail: guirmg@ig.com.br

\begin{abstract}
Introduction: Identify clinical and dosimetric variables involved recurrence, toxicity and overall survival of patients with gastric adenocarcinoma treated with surgery and adjuvant chemoradiotherapy according to protocol INT-0116.

Methods: Patients treated in AC Camargo Cancer Center between 2001-2008 with R0 resection and 3-D conformal radiotherapy were included. Survival, local control, distant metastases, acute and late toxicity were correlated to clinical and dosimetric parameters through univariate and multivariate analyses.

Results: Median follow-up was 42 months, 71 patients were included and $80 \%$ underwent D2 lymphadenectomy. The average number of dissected nodes was 33. 69\% of patients completed treatment according to the protocol. Actuarial-5year overall survival was $57.3 \% .28 .2 \%$ and $15.5 \%$ of the patients had acute toxicity $\geq 3$ and developed late toxicity, respectively. Pathological staging and location in gastroesophageal transition or cardia were correlated to worse survival, whereas pathological staging, vascular invasion and lymphatic invasion to worse local control. Patients who failed to conclude protocol showed higher late toxicity.
\end{abstract}

Conclusions: Patients who underwent R0 resection and D2 lymphadenectomy treated according to Protocol INT-0116 with 3-D conformal radiotherapy have reasonable overall survival and local control with moderate toxicity profile. Prognostic factors involved in recurrence, survival and toxicity were found.

2. Keywords: Gastric cancer; Gastroesophageal junction cancer; Adjuvant chemo-radiotherapy

\section{Introduction}

Adenocarcinomas of the stomach and Gastroesophageal Transition (GET) account for the second cause of death from cancer worldwide. The world incidence of these tumors is of approximately $1,000,000$ of new cases per year. In western countries 5 -year survival of patients suffering from this disease is only about $20-30 \%(1-3)$.

Surgery is an essential part of treatment but, when performed alone, it may have high rates of local and distant failures. After surgery alone overall survival in 5 years is $34-70 \%$ in stages I and II and $7-20 \%$ in stages III and IV, even after R0 resection and D1 or D2 lymphadenectomy (4-8).

Adjuvant radio-chemotherapy was an important progress in the treatment of denocarcinomas of the stomach and GET. The study of the Southwest Oncology Group / Intergroup 0116 (INT-0116) published in 2001 was the first major randomized prospective study to show the benefits of radiochemotherapy in Local Control (LC) (9). Recent update of this study, now with more than 10 years of median follow-up, keeps the increase of the overall survival and progression-free survival rates at the expenses of acceptable rates of late side effects and second tumors (10). INT-0116 suffered some criticism, such as the fact that most patients have been subjected to suboptimal lymphadenectomies, which could have overestimated the actual benefit of radiochemotherapy.

We report our experience with a series of patients operated for adenocarcinoma of the stomach and Gastroesophageal Transition (GET), most of them subject to D2 lymphadenectomy, who later underwent adjuvant treatment according to protocol INT-0116 with 3-D Conformal Radiotherapy (3D-CRT) at a large, multidisciplinary, tertiary care cancer center, and we tried to identify clinical and dosimetric variables involved in the recurrence pattern, toxicity profile and Cancer Specific Survival (CSS). This study was approved by AC Camargo Cancer Center's Ethics Committee.

\section{Methods}

\section{Patients' Characteristics}

Between January 2001 and December 2008, 71 patients 
underwent surgery with curative purpose and R0 resection for adenocarcinoma of the stomach or GET followed by radiochemotherapy with 3D-CRT technique at AC Camargo Cancer Center. The assessment before starting the treatment included medical history, physical examination, blood tests, X-ray or tomography of the chest and tomography of abdomen and pelvis. All patients selected in this study started adjuvant treatment within 90 days after the surgical approach. The eligibility criteria used were similar to those of INT-0116: staging according to the International Union Against Cancer (UICC) IB to IV (M0), adenocarcinoma histology and performance status lower than or equal to 2 (9).

The median follow-up observed was of 42 months (5-112 months). Median age was 61 years (24-86 years), $73.2 \%$ of the patients were of the male gender and $33.8 \%$ of the patients had signet ring histology. According to the $7^{\text {th }}$ edition of the American Joint Commission on Cancer, $5.6 \%$ were staged as IB, $15.5 \%$ IIA, 35.2\% IIB, 19.7\% IIIA, 9.8\% IIIB, 12.7\% IIIC and 1.4\% were incompletely staged (Table 1 Patient Characteristics).

\section{Characteristics of Treatment}

The surgical treatment used varied according to the location and extension of the primary tumor. The surgery consisted of total or partial gastrectomy with circumferential margin of $5 \mathrm{~cm}$ when technically feasible, associated with D1 or D2 lymph node dissection. In our series, $80 \%$ of the patients underwent D2 dissection and the average number of lymph nodes removed was 33 (6-84 lymph nodes).

Chemotherapy consisted of Fluorouracil (5-FU) at the dose of $425 \mathrm{mg}$ per square meter of body surface, every day, and Leucovorin, $20 \mathrm{mg}$ per square meter daily, during 5 days. Twenty eight days after the initial cycle of chemotherapy, radiochemotherapy started with a dose of 45 Gy at daily fractions of 180 cGy, 5 days a week, during 5 weeks, simultaneously with chemotherapy using 5-FU at the dose of $400 \mathrm{mg}$ per square meter a day and Leucovorin $20 \mathrm{mg}$ per square meter a day for the first 4 days and last 3 days of radiation therapy. One month after the conclusion of the radiation therapy, 2 cycles of 5 days with 5-FU at the dose of $425 \mathrm{mg}$ per square meter a day, and Leucovorin 20 mg per square meter a day, were offered separately for a period of 1 month.

During radiotherapy simulation, patients were immobilized in supine position using a vacuum immobilizer. The treatment volume was defined in accordance with INT-0116 and included the stomach and tumor bed, regions of anastomosis and regional lymph nodes, according to the location and staging. Upper gastrointestinal endoscopy tests, endoscopic ultrasound and Computed Tomography (CT) were used to define the location of the primary tumor and its relation with the nodal stations. All planning was made with 3D-CRT and the treatment was planned with 4 photon beams in a linear accelerator with energy of 6,10 or 15 MV. The Planning Target Volume (PTV) was created from the Clinical Target Volume (CTV) plus $1 \mathrm{~cm}$ margins in all directions and portal images were made weekly to check positioning. The average PTV volume obtained in this work was 980 cc (544-2273 cc).

\begin{tabular}{|l|l|}
\hline \multicolumn{2}{|l|}{ Table 1: Patient Characteristics (71 patients). } \\
\hline Median Follow Up & $\mathbf{4 2}$ months (5-112) \\
\hline Pathological Staging & $5.6 \%$ IB $\quad 19.7 \%$ IIIA \\
& $15.5 \%$ IIA $\quad 9.8 \%$ IIIB \\
& $35.2 \%$ IIB $\quad 12.7 \%$ IIIC \\
\hline Median Age & 61 incompletely staged \\
\hline Signet Ring Histology & $33.8 \%$ \\
\hline Male Gender & $73.2 \%$ \\
\hline D2 lymhadenectomy & $80 \%$ \\
\hline $\begin{array}{l}\text { Dissected Nodes } \\
\text { (average number) }\end{array}$ & $33(6-84)$ \\
\hline Protocol Conclusion & $69 \%$ \\
\hline PTV(average volume) & $980 \mathrm{cc}(544-2273)$ \\
\hline
\end{tabular}

\section{Outcome Assessment}

There was one appointment every week during radiochemotherapy and, subsequently, patients went to see at least one of the physicians, at 3-6 month interval, from the departments of Abdominal Surgery, Radiation Oncology or Clinical Oncology at AC Camargo Cancer Center for 5 years and, then, every year. Toxicity was assessed from clinical visit documentation and graded using the NCI Common Toxicity Criteria for Adverse Events (CTCAE - v.3.0) and the highest grade given at any time point was used in the analysis. CT scan of the abdomen and pelvis or Magnetic Resonance Imaging (MRI) of the pelvis was obtained at each 3 to 6 month interval. Carcinoembryonic Antigen (CEA) values were obtained based on physician preference. Follow-up was calculated from the date of surgery.

Recurrences were categorized as locoregional or distant. Locoregional recurrence was defined as relapse in the stomach bed, regional lymph nodes or in the region of anastomosis. Peritoneal carcinomatosis was included as locoregional relapse. Distant metastases were defined as metastasis in the liver, lung or other organs (bones, brain, and ovaries). Distant failure was reported in view of the location in which it was documented the first site of relapse, obtained most of the time through radiological evidence or operative findings with biopsy.

\section{Statistical analysis}

All statistical calculations were made using software SPSS 13.0. The survival probabilities were estimated using the KaplanMeier method, and groups were compared using a log-rank test $(11,12)$. All tests were two-sided and $p \leq 0.05$ was considered statistically significant.

Selected clinical and dosimetric parameters were investigated by univariate analysis. Variables with p-value $\leq 0.1$ in the univariate analysis were used to build the multivariate model. Multivariate analysis of the data was performed using the Cox proportional hazards model (13). 


\section{Results}

\section{Overall Survival, Cancer Specific Survival, and Recurrence Rates}

The OS in 5 years was $57.3 \%$ (Figure 1 Actuarial overall survival) and CSS in 5 years was $64.6 \%$ (Figure 2 Actuarial cancer specific survival). Statistically significant factors associated with worse CSS included pathological staging (Figure 3 Pathological staging and actuarial cancer specific survival) and location in GET or cardia (Table 2 Local control and distant metastasis univariate analysis) (Table 3 Multivariate analysis - Local control, toxicity and survival).

Location in GET or cardia was the only factor associated with higher risk of distant metastasis $(P=0.004)$ (Table 2 and 3). Pathological staging, vascular and lymphatic invasion were factors associated with worse locoregional control in the multivariate analysis (Table 3). The 5-year cumulative incidence

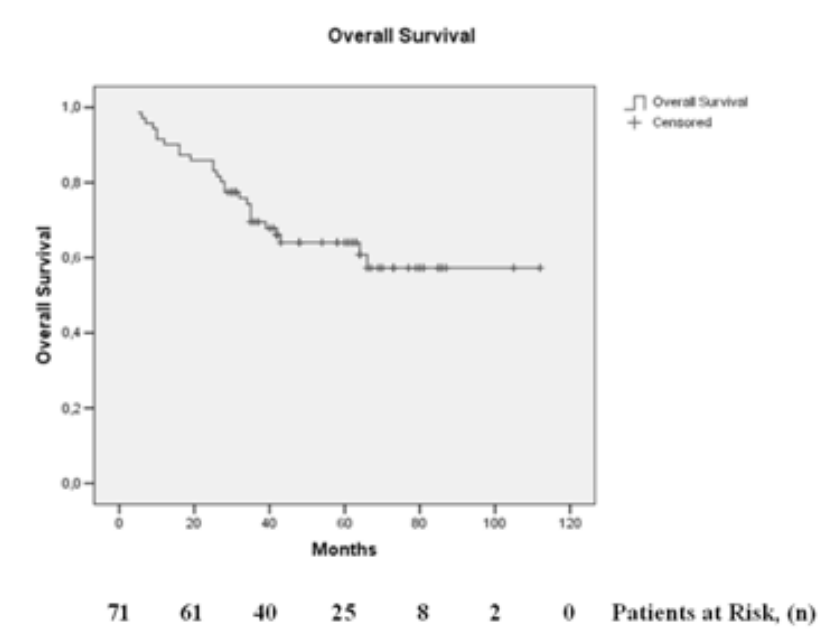

Figure 1: Actuarial Overall Survival.

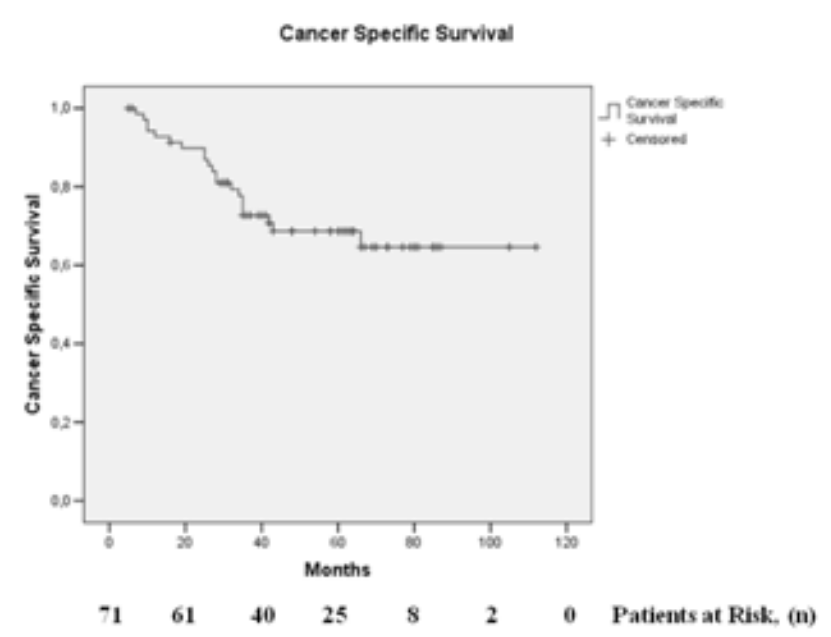

Figure 2: Actuarial Cancer Specific Survival.

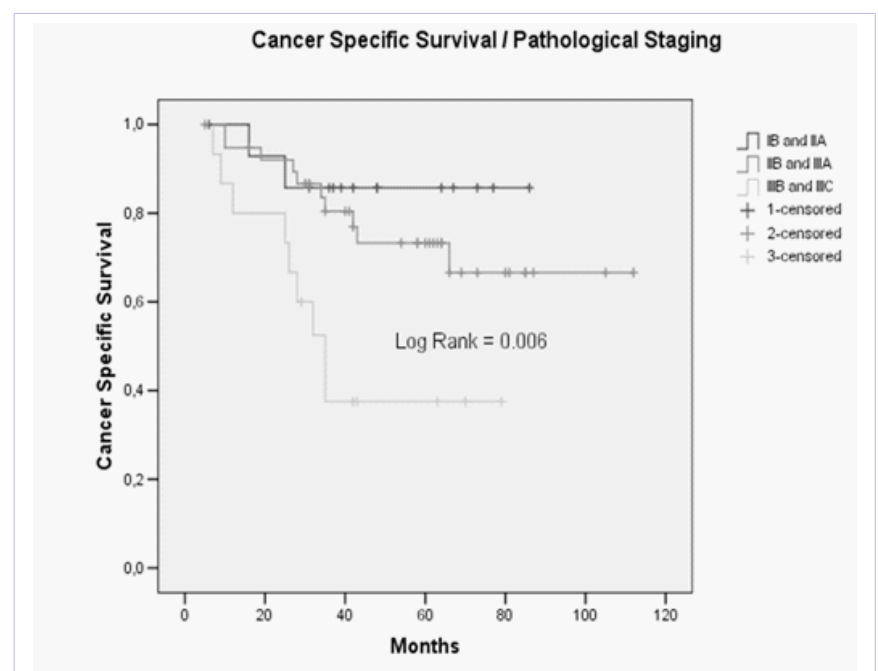

Patients at Risk, (n)

\begin{tabular}{|c|c|c|c|c|c|}
\hline IB, IIA: $\quad 15$ & 13 & 8 & 5 & 1 & 0 \\
\hline IIB, IIA: 39 & 34 & 25 & 17 & 7 & \\
\hline IIIB, IIIC: 15 & 12 & 5 & 3 & 0 & 0 \\
\hline
\end{tabular}

Figure 3: Pathological staging and actuarial cancer specific survival.

of distant metastasis was $24 \%$ (17 of 71 patients treated), $5.6 \%$ ( 4 of 71 patients) had local and distant failure, and 25.3\% (18 of 71 patients) had locoregional recurrence.

\section{Toxicity}

Twenty patients (28.2\% of the 71 patients) developed acute toxicity rated as higher than or equal to level 3 according to CTCAE 3.0, and $12.6 \%$ of the patients suffered from late side effects, defined as symptoms presented 6 months after radiochemotherapy. Forty-nine patients $(69 \%$ of the 71 patients treated) concluded the treatment according to protocol. Patients with toxicity higher than or equal to 3 according to CTCAE 3.0 had gastrointestinal or hematological side effects in $47.3 \%$ and $22.2 \%$ of the times, respectively, whereas mucositis and fatigue occurred in $16.6 \%$ and $13.6 \%$ of the times, respectively.

Nine patients (12.6\% of the 71 patients treated) suffered from late side effects such as epigastralgia (4 patients), anastomotic stricture ( 3 patients), intestinal fistula ( 1 patient) and pancreatic insufficiency ( 1 patient). The incompleteness of the treatment was related to a higher risk of developing late toxicity ( $p=$ 0.008). Female gender and PTV volume higher than $1150 \mathrm{cc}$ were parameters associated with gastrointestinal toxicity higher than or equal to 3 in the univariate analysis, but not in the multivariate analysis ( $p=0.19$ and 0.13 , respectively) (Tables 2 and 3 ).

\section{Discussion}

Adenocarcinomas of the stomach and GET are challenging tumors, with reserved prognosis when treated with surgery alone $(1-3,8)$. Locoregional failures of $38-87.8 \%$ were identified in clinical and autopsy series after surgical resection alone. $(6,7)$. In this study, locoregional recurrence rates of $30.9 \%$ were observed 

Radiotherapy

\begin{tabular}{|c|c|c|}
\hline Univariate Analysis & $\begin{array}{l}\text { LOCAL CONTROL* } \\
\text { (Significance Level) }\end{array}$ & $\begin{array}{c}\text { DISTANT } \\
\text { METASTASIS } \\
\text { (Significance Level) }\end{array}$ \\
\hline Age & 0.67 & 0.90 \\
\hline Gender & 0.74 & 0.58 \\
\hline Pathological Staging & 0.007 & 0.65 \\
\hline Grade & 0.12 & 0.18 \\
\hline $\begin{array}{l}\text { Location In GET or } \\
\text { Cardia }\end{array}$ & 0.65 & $\begin{array}{l}0.004 \text { (worse if GET or } \\
\text { cardia }+ \text { ) }\end{array}$ \\
\hline Type of Surgery & $\begin{array}{l}0.008 \text { (better with } \\
\text { subtotal gastrectomy) }\end{array}$ & 0.08 \\
\hline Protocal Conclusion & 0.25 & 0.14 \\
\hline Lymphatic Invasive + & 0.03 & 0.67 \\
\hline Vascular Invasive + & 0.01 & 0.22 \\
\hline Perineural Invasive + & 0.09 & 0.18 \\
\hline
\end{tabular}

Table 3: Multivariate Analysis - Local Control, Toxicity and Survival.

\begin{tabular}{|c|c|}
\hline Multivariate Analysis & LOCAL CONTROL \\
\hline Pathological Staging & 0.004 \\
\hline Type of Surgery & 0.20 \\
\hline Lymphatic Invasive + & 0.04 \\
\hline Vascular Invasive + & 0.01 \\
\hline Multivariate Analysis & G3 GASTROINTESTINAL ACUTE \\
TOXICITY
\end{tabular}

*PTV Volume (p50, p75 and p90) means PTV Volume in percentiles 50 $\%, 75 \%$ and $90 \%$, respectively

with the implementation of radiochemotherapy associated most of the times with D2 lymphadenectomy, which can be favorably compared to the previously described series. It is important to consider that, in addition to radiochemotherapy, factors that help improve the outcomes also include an improvement in preoperative staging and in surgical techniques for the past ten years, as well as the execution of D2 lymphadenectomy in $80 \%$ of the cases.

Systemic control of the disease is still a problem in GET and stomach cancer. Distant failures occurred in $29.5 \%$ of the patients in this study, which can be compared to those observed in other works (4). Several publications with new strategies of perioperative chemotherapy treatment, concurrent to radiation therapy or in the adjuvant context have been made at the attempt of optimizing the control of the distant disease. Agents such as Taxanes, Oxaliplatin, Capecitabine and Trastuzumab are used in the treatment of these tumors and their association and timing need to be better investigated in treatment within the curative context (14). Besides the radiochemotherapy scheme established by INT-0116, other strategies also demonstrated an improvement in the survival of patients with gastric adenocarcinomas. The MAGIC trial demonstrated that preoperative chemotherapy with 3 cycles of ECF (epirubicin, cisplatin and 5-FU in continuous infusion) offered before and after surgery significantly increased the overall survival of these patients in 5 years, going from $23 \%$ to $36.3 \%$ (24). A Japanese prospective randomized study, after gastrectomy with D2 dissection, demonstrated that adjuvant chemotherapy with S-1 improved OS in 3 years, going from $70.1 \%$ to $80.1 \%$ (25).

The optimal number of lymph nodes which must be dissected is still a topic of controversy in the treatment of gastric cancer. There is a recommendation of the American Joint Commission on Cancer suggesting that it is necessary the analysis of 15 or more lymph nodes for accurate staging (15). An analysis from SEER of 1988-2001 showed that 15 lymph nodes were examined in only $27.6 \%$ of the cases (16) and that better survival was observed in these patients $(17,18)$. There are other studies that suggest that the removal of 25 lymph nodes is beneficial to survival $(17,18)$; however, western studies have never demonstrated the role of extended lymph node dissection in the increase of overall survival (19-21).

The update of INT-0116, currently with median followup longer than 10 years, demonstrated that the benefits of radiochemotherapy in the increase of OS and of locoregional disease control are kept in the long term, increasing overall survival and reducing the risk of relapse in $32 \%$ and 52\%, respectively, when compared to patients treated with surgery alone (10). Initially this study was criticized because the vast majority of patients underwent suboptimal lymphadenectomy, and $54 \%$ of the patients underwent gastrectomy with D0 dissection, leading to the hypothesis that postoperative radiochemotherapy might have compensated for a suboptimal surgery (9).

Further evidence which support the use of radiochemotherapy in Adenocarcinomas of the stomach or GET in the adjuvant context is, for instance, meta-analysis with 708 patients suffering from gastric cancers which demonstrated that adjuvant radio-chemotherapy decreased the mortality rate in 5 years in $55 \%$ when compared to surgery alone (22) and a Korean observational study comparing surgery alone with D2 dissection versus this same kind of surgery with postoperative radio-chemotherapy (5-y OS, $57.1 \%$ vs. $51 \%, p=0.02$ ) (23).

In our study, data analysis is limited for its retrospective nature and due to the fact that there is no group available for comparison which did not receive radiochemotherapy. Moreover, the recurrence pattern was determined from the analysis of data verified in the medical history, which can underestimate the actual rates of locoregional failures. In spite of these limitations, patients in this work received treatment in a relatively uniform manner and, even with the poor prognosis associated with stomach and GET neoplasms, it was possible to obtain OS rates in 5 years of $57.3 \%$ and cancer specific survival in 5 years of $64.6 \%$, 
not being possible to achieve median survival with median follow-up of 42 months.

Unfortunately, acute toxicity in the treatment proposed by INT-0116 is high. Acute toxicities in grade 3 or 4 of $41 \%$ and $32 \%$, respectively, were reported in the original work (9) in which all patients were treated with two-dimensional planning techniques and mainly with parallel opposed fields. The use of 3D-CRT in our series did not demonstrate clear improvement in acute toxicity rates, and $31 \%$ of the patients were unable to complete treatment due to toxicity. Another limitation of this work is the assessment of toxicity based on information gathered in medical histories and which, therefore, has limitations due to the subjective nature of the report made by different patients and interpretation made by assistant physicians. In INT-0116, 65\% of the patients finished treatment as planned, even using conventional radiation therapy, and similar results were demonstrated in series of Australia, Korea and Singapore (26-28). Analyzing late toxicity rates due to radiochemotherapy is complex because they get mixed up with the late side effects from the surgical approach and because they have multifactorial origin. In this series, patients who had local failure were excluded from the analysis of late toxicity since it is unfeasible to define whether the mentioned symptoms resulted from prior treatment or from tumor progression itself. The incidence of late side effects observed in this series was acceptable and comparable with the few reports available in literature regarding late symptoms of adjuvant radio-chemotherapy in gastric cancer (20).

In conclusion, patients in this series who underwent R0 resection and D2 lymphadenectomy treated according to Protocol INT-0116 with 3D-CRT have reasonable and comparable to literature data OS, CSS and LC rates with moderate toxicity profile. Pathological staging and location in GET or cardia were factors with impact on CSS. Vascular invasion, lymphatic invasion and pathological staging were predictors of worse LC, whereas location in GET or cardia was the sole parameter correlated to DM. Patients who failed to conclude the protocol showed higher late toxicity.

\section{References}

1. Bonenkamp JJ, Hermans J, Sasako M, van de Velde CJ, Welvaart K, Songun I, et al. Extended lymph-node dissection for gastric cancer. N Engl J Med. 1999; 340(12):908-14.

2. Landry J, Tepper JE, Wood WC, Moulton EO, Koerner F, Sullinger J. Patterns of failure following curative resection of gastric carcinoma. Int J Radiat Oncol Biol Phys. 1990; 19(6):1357-62.

3. Cuschieri A, Weeden S, Fielding J, Bancewicz J, Craven J, Joypaul V, et al. Patient survival after D1 and D2 resections for gastric cancer: long-term results of the MRC randomized surgical trial. Surgical Cooperative Group. Br J Cancer. 1999; 79(9-10):1522-30.

4. Quero L1, Bouchbika Z, Kouto H, Baruch-Hennequin V, Gornet JM, Munoz N, et al. Postoperative Chemotherapy Followed by Conformal Concomitant Chemoradiotherapy in High-Risk Gastric Cancer. Int J Radiat Oncol Biol Phys. 2012; 83(2):574-80. doi: 10.1016/j. ijrobp.2011.07.031.

5. Gunderson LL. Gastric cancer patterns of relapse after surgical resection. Semin Radiat Oncol. 2002; 12(2):150-61.
6. Gunderson LL, Sosin H. Adenocarcinoma of the stomach: Areas of failure in a re-operation series (second or symptomatic look) clinicopathologic correlation and implications for adjuvant therapy. Int J Radiat Oncol Biol Phys. 1982; 8(1):1-11.

7. Mcneer g, Vandenberg h jr, Donn fy, Bowden l. A critical evaluation of subtotal gastrectomy for the cure of cancer of the stomach. Ann Surg. $1951 ; 134(1): 2-7$.

8. Hundahl SA, Phillips JL, Menck HR. The National Cancer Data Base Report on poor survival of U.S. gastric carcinoma patients treated with gastrectomy: Fifth Edition American Joint Committee on Cancer staging, proximal disease, and the "different disease" hypothesis. Cancer 2000; 88(4):921-32.

9. Macdonald JS, Smalley SR, Benedetti J, Hundahl SA, Estes NC, Stemmermann GN, et al. Chemoradiotherapy after surgery compared with surgery alone for adenocarcinoma of the stomach or gastroesophageal junction. N Engl J Med. 2001; 345(10):725-30.

10. Smalley SR, Benedetti JK, Haller DG, Hundahl SA, Estes NC, Ajani JA, et al. Updated Analysis of SWOG-Directed Intergroup Study 0116: A Phase III Trial of Adjuvant Radiochemotherapy Versus Observation After Curative Gastric Cancer Resection. J Clin Oncol. 2012; 30(19):2327-33. doi: 10.1200/JCO.2011.36.7136.

11. Kaplan EL, Meier P. Non-parametric estimation for incomplete observations. J Am Stat Assoc. 1958; 53:457-481.

12. Peto R, Peto J. Asymptotically efficient rank invariant procedures (with discussion). J Royal Stat Soc 1972; 135:185-207.

13. Cox D. Regression models and life tables. J R Stat Soc B 1972; $34(2): 187-220$.

14. Douglass HO Jr, Nava HR. Gastric adenocarcinoma: Management of the primary disease. Semin Oncol. 1985; 12(1):32-45.

15. L.H. Sobin, Ch. Wittekind. International Union against Cancer. TNM classification of malignant tumors. 6th ed. New Jersey. John Wiley \& Sons; 2002.

16. Coburn NG, Swallow C, Quan ML, Law C. Significant regional variation in treatment and survival of gastric cancer. [Abstract]. Proc Am Soc Clin Oncol 2005; 23(Suppl. 16S):4004.

17. Marubini E, Bozzetti F, Miceli R, Bonfanti G, Gennari L, et al. Lymphadenectomy in gastric cancer: Prognostic role and therapeutic implications. Eur J Surg Oncol. 2002; 28(4):406-12.

18. Scartozzi M1, Galizia E, Graziano F, Catalano V, Berardi R, Baldelli AM, et al. Over-DI dissection may question the value of radiotherapy as a part of an adjuvant programme in high-risk radically resected gastric cancer patients. Br J Cancer. 2005; 92(6):1051-4.

19. Hartgrink HH, van de Velde CJ, Putter H, Bonenkamp JJ, Klein Kranenbarg E, Songun I, et al. Extended lymph node dissection for gastric cancer: Who may benefit? Final results of the randomized Dutch gastric cancer group trial. J Clin Oncol. 2004; 22(11):2069-77.

20. Kassam Z, Lockwood G, O’brien C, Brierley J, Swallow C, Oza A, et al. Conformal Radiotherapy in the adjuvant treatment of gastric cancer: overview of 82 cases. Int J Radiat Oncol Biol Phys. 2006; 65(3):713-9.

21. Degiuli M, Calvo F. Survival of early gastric cancer in a specialized European center: Which lymphadenectomy is necessary? World J Surg. 2006; 30(12):2193-203.

22. Fiorica F, Cartei F, Enea M, Licata A, Cabibbo G, Carau B, et al. The impact of radiotherapy on survival in resectable gastric carcinoma: a meta-analysis of literature data. Cancer Treat Rev. 2007; 33(8):72940 . 


\section{Radiotherapy}

23. Kim S, Lim DH, Lee J, Kang WK, MacDonald JS, Park CH, et al. An observational study suggesting clinical benefit for adjuvant postoperative chemoradiation in a population of over 500 cases after gastric resection with D2 nodal dissection for adenocarcinoma of the stomach. Int J Radiat Oncol Biol Phys. 2005; 63(5):1279-85.

24. Cunningham D, Allum WH, Stenning SP, Thompson JN, Van de Velde CJ, Nicolson M, et al. Perioperative chemotherapy versus surgery alone for resectable gastroesophageal cancer. N Engl J Med. 2006; 355(1):11-20.

25. Sasako M, Sakuramoto S, Katai H, Kinoshita T, Furukawa H, Yamaguchi T, et al. Five-year outcomes of a randomized phase III trial comparing adjuvant chemotherapy with S-1 versus surgery alone in stage II or III gastric cancer J Clin Oncol. 2011; 29(33):4387-93. doi: 10.1200/ JC0.2011.36.5908.
26. Back MF, Premsenthil S, Wynne CJ, Shakespeare TP, et al. Introducing the Intergroup 0116 protocol of adjuvant chemo-radiotherapy in gastric cancer into clinical practice. Clin Oncol (R Coll Radiol). 2003; 15(7):378-82.

27. Hughes BG, Yip D, Chao M, Gibbs P, Carroll S, Goldstein D, et al. Audit of postoperative chemoradiotherapy as adjuvant therapy for resected gastroesophageal adenocarcinoma: An Australian multicentre experience. ANZ J Surg. 2004; 74(11):951-6.

28. Park SH, Kim DY, Heo JS, Lim DH, Park CK, Lee KW, et al. Postoperative chemoradiotherapy for gastric cancer. Ann Oncol. 2003; 14(9):13737. 